\title{
Neues von der Palmengarten-Gesellschaft
}

Die Palmengarten-Gesellschaft „Freunde des Palmengartens e. V. “ berichtet über ein weiteres sehr erfolgreiches Jahr.

Die Förderung des Palmengartens erreichte 2007 mit ca. $117000 €$ eine Rekordhöhe. Größter Einzelposten darin war die Erneuerung des Rosengartens mit ca. $77000 €$. Am 2. November 2007 wurden am Haus Rosenbrunn unter Mitwirkung der Stadträtin Manuela Rottmann und der Patin des Rosengartens, Franziska ReichenBACHER, die ersten Rosen gepflanzt. Darüber ist in den Medien ausführlich berichtet worden.

Die Zahl unserer Mitglieder stieg innerhalb eines Jahres von ca. 1700 auf über 2000!

An der Mitgliederversammlung am 23. April 2008 nahmen 173 Mitglieder teil. Eine zuvor nie erreichte Anzahl, die den Siesmayer-Saal an die Kapazitätsgrenze brachte.

2007 verstarben 20 Mitglieder, darunter unser Ehrenmitglied KLÄre SchneIder (50 Jahre Mitglied) und Wolfgang Claus (in 2007 60jährige Mitgliedschaft).

Mit der Goldenen Ehrennadel der Gesellschaft wurden für langjährige Mitgliedschaft geehrt: Eckhard Beutnagel (50 Jahre), Tilly Jöckel, Helmut Otтo Schwab und Gattin (jeweils 40 Jahre). Der langjährige Vorsitzende der Gesellschaft, Professor Dr. Georg ZizKa, wurde zum Ehrenmitglied gewählt.

Herta Menk, seit 30 Jahren Mitglied, seit 1985 Mitglied des Vorstands und seit 2000 Schatzmeisterin, gab ihr Amt in jüngere Hände. Sie verantwortet weiterhin die Tagesfahrten und Mehrtagesreisen und bleibt Mitglied des erweiterten Vorstandes. Für ihre langjährigen Verdienste wurde Herta Menk mit der Goldenen Ehrennadel ausgezeichnet und zum Ehrenmitglied gewählt. Zum Abschied wurde eine Buddleja 'Nanho Blue' für ihren Garten überreicht.
Lolita Sebastian, seit einem Jahr Mitglied des erweiterten Vorstands, wurde als Nachfolgerin von Herta Menk als Schatzmeisterin in den Vorstand berufen. Frau Sebastian ist durch ihre Ausbildung (Bankkauffrau, Betriebswirtin) und berufliche Erfahrung (langjährig im Kreditgeschäft tätig, Abteilungsdirektorin einer deutschen Geschäftsbank) sowie nach der Einarbeitung durch die ausscheidende Schatzmeisterin bestens für diese Aufgabe vorbereitet.

Die Mitglieder des Helferkreises um DoritT H. Röhnert wurden bei der Mitgliederversammlung vorgestellt und erhielten einen Sonderapplaus für die erfolgreiche Mitgliederwerbung. Wenn Sie auch im Helferkreis mitmachen möchten, melden Sie sich bitte bei der Geschäftsstelle. Das Internet wird zunehmend von unseren Mitgliedern genutzt, um an Informationen über die Palmengartengesellschaft zu gelangen. Wir wollen künftig eine schnellere und kostengünstigere Kommunikation mit unseren Mitgliedern pflegen. Nehmen auch Sie daran teil und schicken Sie Ihre E-Mail-Adresse an die Geschäftsstelle unter info@palmengartengesellschaft.de. Sie helfen mit, Kosten (und Papier!) zu sparen und der Geschäftsstelle die Arbeit zu erleichtern. Wir haben bereits über 270 E-Mail-Adressen verzeichnet. Auch unsere Homepage wird immer wichtiger. Eine kleine Gruppe von Mitgliedern ist bereit, uns bei der Aktualisierung und dem Ausbau ehrenamtlich zur Seite zu stehen. Sie dürfen gespannt sein. Darüber hinaus wurde aus dem Mitgliederkreis Hilfe angeboten, uns bei Steuerberatungs- und Rechtsfragen ehrenamtlich zu unterstützen.

Unser Veranstaltungsprogramm 2007 war wieder sehr vielfältig und umfangreich. Angeboten wurden 9 Vorträge (organisiert von Herbert Billensteiner), 12 Führungen (Ditmar BreimHORST), 11 Veranstaltungen „Pflanze des Monats" (Dr. Hilke Steinecke), das bewährte Schüler-Seminar (Ute Köllen, Astrid Steindorf, Beate Vaupel) sowie Tages- und Mehr- 


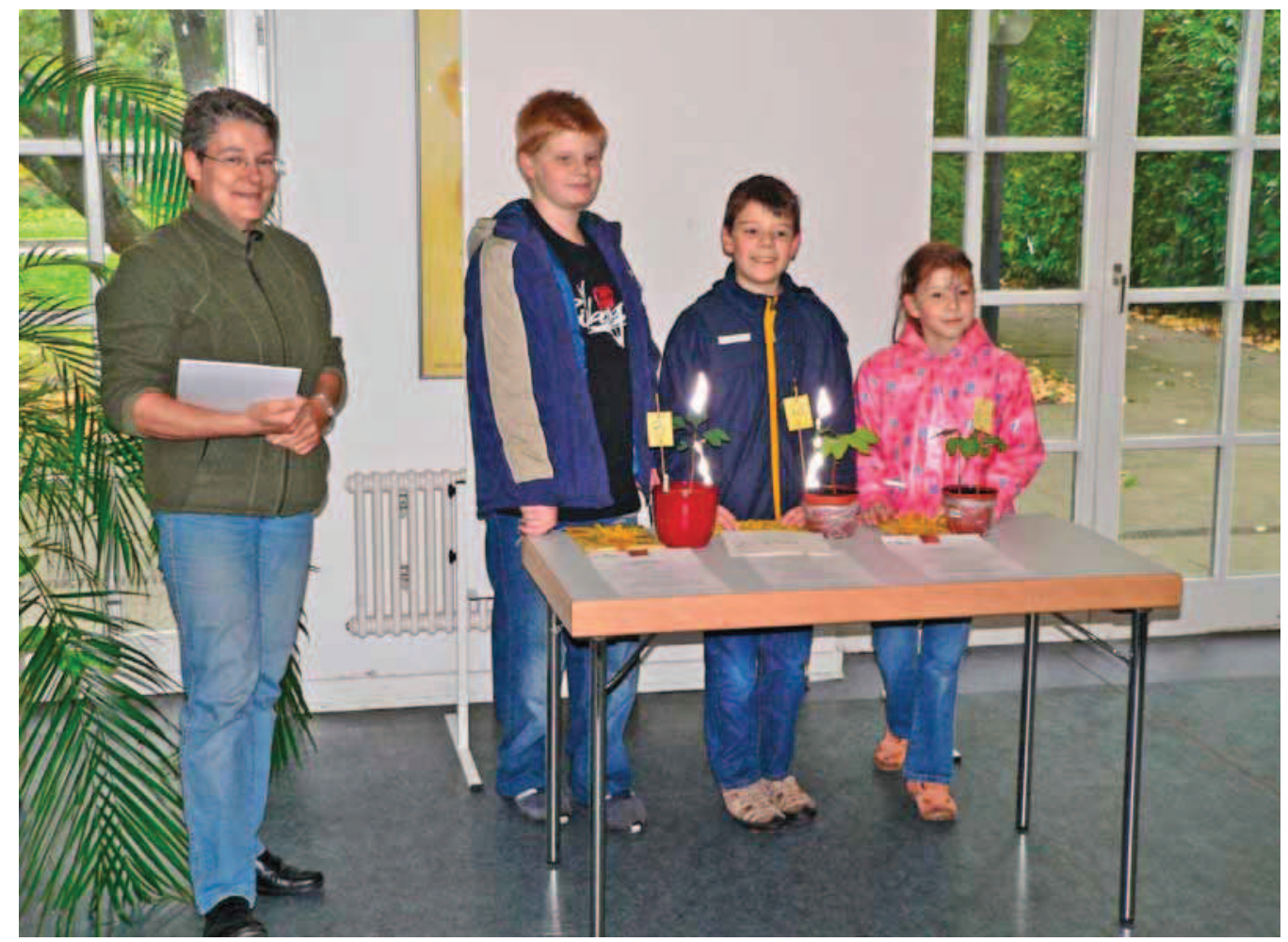

tagesreisen (Herta MenK). Insgesamt nahmen an diesen Veranstaltungen über 1300 Personen teil, darunter auch viele Besucherinnen und Besucher, die dafür eigens in den Palmengarten kamen. Viele davon sind inzwischen Mitglied unserer Gesellschaft geworden. Am Schülerseminar nahmen über 30 Kinder zwischen 5 und 12 Jahren teil. Thema 2007: „Bäume“. Von April bis Oktober (6 Samstage) wurden die jungen Teilnehmerinnen und Teilnehmer mit den Bäumen im Palmengarten vertraut gemacht. Die erfolgreiche Betreuung der Sämlinge aus der Palmengarten-Gärtnerei zu kleinen Bäumchen wurde durch Urkunden belohnt, die begeistert aufgenommen wurden. 2008 lautet das Thema „Duft“. Wir sind heute schon auf das Ergebnis gespannt. Ziele der Tagesreisen (Dr. Astrid JaCOBS) waren 2007 Rodheim und Kronberg sowie Windesheim und Bad Sobernheim. 2008 stehen Ladenburg und Weinheim

Abb. 1: Beate Vaupel mit den Preisträgern des Schülerseminars.
(31. Mai) sowie Bingen (26. Juli) auf dem Programm. Die Mehrtagesreise 2007 (Ruge-Reisen) führte zu den Schlössern und Gärten der Loire. 2008 führt sie nach Belgien. Nähere Auskünfte erteilt die Geschäftsstelle.

2007 wurden erstmalig Praxis-Seminare (Helga Urban) zu den Themen „Das Schneiden von Rosen“ (3 Termine) und „Das Pflanzen von Rosen“ (1 Termin) angeboten. Aufgrund des großen Erfolges werden diese auch 2008 durchgeführt. Neu im Programm ist eine Veranstaltung zum Thema Orchideen (Herbert BillenSTEINER).

Das Weihnachtstreffen am 4. Dezember 2007, mit musikalischer Begleitung durch unser Ehrenmitglied Heinz Marx (Klavier) und Dr. WenZEL (Violine), war wieder ein voller Erfolg. Herzlicher Dank der KfW-Bankengruppe für die Zurverfügungstellung der großzügigen Räumlichkeiten, dem Café Siesmayer für die Spende des Kuchens sowie Dr. Hilke Steinecke für 


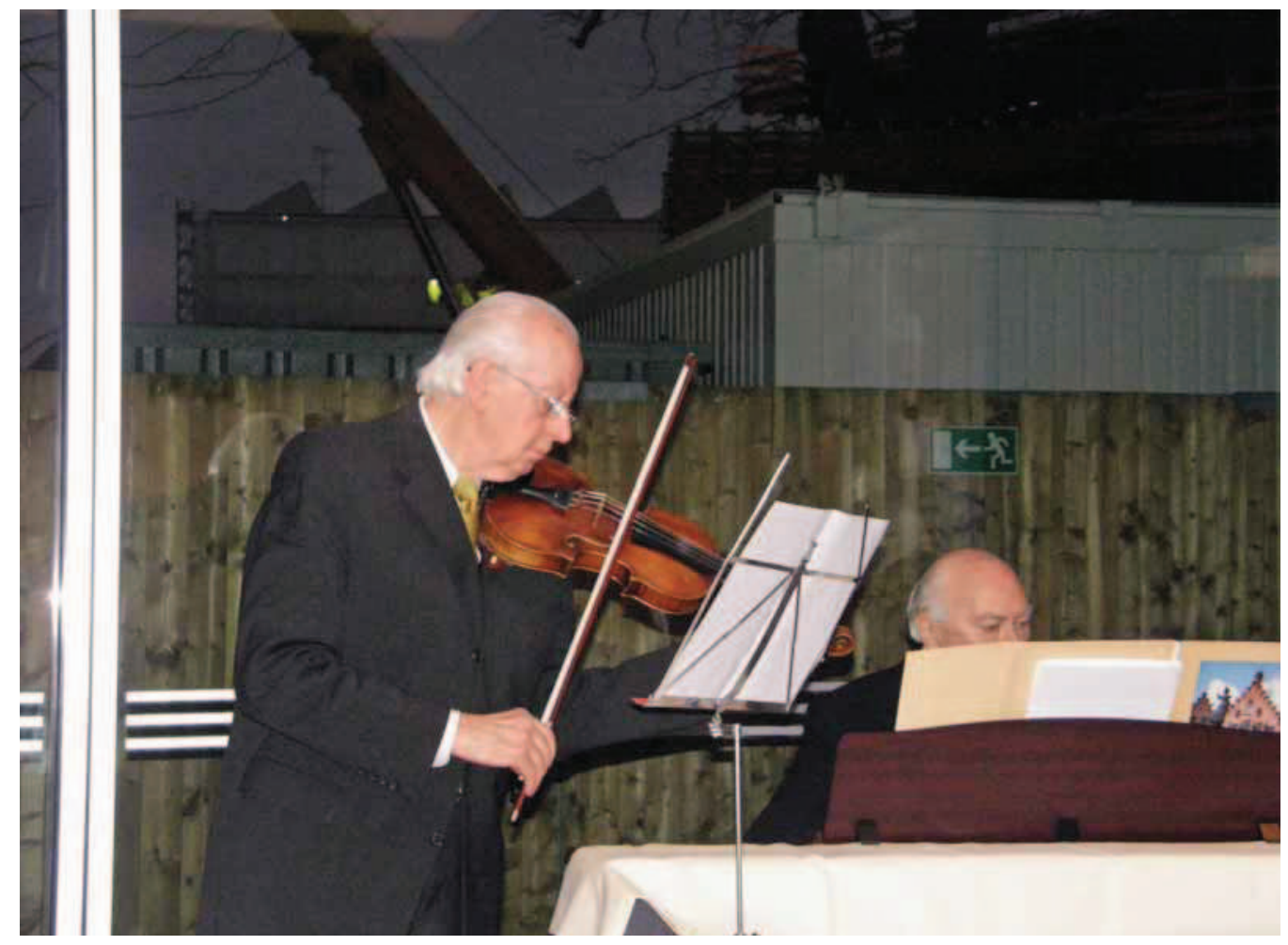

einen großartigen Bilderreigen. Wir konnten glücklicherweise alle Gekommenen unterbringen (Saalkapazität aus feuerpolizeilichen Gründen max. 300 Personen). Unschön ist jedoch, dass über 90 angemeldete Mitglieder nicht kamen. Wer sich nicht abmeldet, nimmt einem anderen Mitglied, das gerne gekommen wäre, den Platz weg. Hier wünschen wir uns ein Umdenken bei den Mitgliedern.

Wir haben für 2008 ein Sommerfest geplant, bei dem es keine Zulassungsbeschränkungen geben wird. Termin ist der 15. Juni 2008. Bewusst haben wir einen Sonntag gewählt, um auch Berufstätigen und Familien mit Kindern die Teilnahme zu ermöglichen. Das Programm umfasst einen musikalischen Frühschoppen am Oktogonbrunnen, ein Nachmittagskonzert am Musikpavillon sowie Aktivitäten für Kinder. Eine gesonderte Einladung folgt.

Die stark gestiegene Zahl der Mitglieder und die Ausweitung der Aktivitäten der Gesellschaft bedeuten auch eine erhebliche Herausforderung für unsere Geschäftsstelle. Hier gebührt Ilse Henning ein ganz besonderer Dank.

Wir stellen uns auf eine weiter steigende Zahl der Mitglieder ein. Wir wollen noch attraktiver werden und damit noch mehr Beiträge und Spenden aufbringen, um die Förderung des Palmengartens noch weiter steigern zu können.

Unseren Mitgliedern wünschen wir ein weiterhin erfolgreiches Jahr.

im Mai 2008

\section{KLaUs Urban \\ - für den Vorstand -}

Abb. 2: Musikalische Untermalung der Weihnachtsfeier für die Mitglieder der Palmengartengesellschaft. 